OPEN ACCESS

Edited by: Jinzhou Huang,

Mayo Clinic, United States

Reviewed by:

Hao Li,

Shanghai Jiao Tong University, China Chenming Wu,

Tongii University, China

*Correspondence:

Yuanliang Yan

yanyuanliang2011@126com

Cong Shen

sc1571062142@qq.com

Specialty section: This article was submitted to Molecular and Cellular Oncology,

a section of the journal

Frontiers in Cell and Developmental

Biology

Received: 09 November 2021 Accepted: 29 November 2021 Published: 13 December 2021

Citation:

Xia F, Yan Y and Shen C (2021) A Prognostic Pyroptosis-Related IncRNAs Risk Model Correlates With the Immune Microenvironment in

Colon Adenocarcinoma.

Front. Cell Dev. Biol. 9:811734. doi: 10.3389/fcell.2021.811734

\section{A Prognostic Pyroptosis-Related IncRNAs Risk Model Correlates With the Immune Microenvironment in Colon Adenocarcinoma}

\author{
Fada Xia ${ }^{1}$, Yuanliang Yan ${ }^{1 *}$ and Cong Shen ${ }^{1,2 *}$ \\ ${ }^{1}$ Center for Molecular Medicine, Xiangya Hospital, Key Laboratory of Molecular Radiation Oncology of Hunan Province, Central \\ South University, Changsha, China, ${ }^{2}$ National Clinical Research Center for Geriatric Disorders, Xiangya Hospital, Central South \\ University, Changsha, China
}

Recent studies have indicated that long non-coding RNAs (IncRNAs) may participate in the regulation of tumor cell proptosis. However, the connection between IncRNA expression and pyroptosis remains unclear in colon adenocarcinoma (COAD). This study aims to explore and establish a prognostic signature of COAD based on the pyroptosis-related IncRNAs. We identify 15 prognostic pyroptosis-related IncRNAs (ZNF667-AS1, OIP5AS1, AL118506.1, AF117829.1, POC1B-AS1, CCDC18-AS1, THUMPD3-AS1, FLNBAS1, SNHG11, HCG18, AL021707.2, UGDH-AS1, LINC00641, FGD5-AS1 and AC245452.1) from the TCGA-COAD dataset and use them to construct the risk model. After then, this pyroptosis-related IncRNA signature is validated in patients from the GSE17536 dataset. The COAD patients are divided into low-risk and highrisk groups by setting the median risk score as the cut-off point and represented differences in the immune microenvironment. Hence, we construct the immune risk model based on the infiltration levels of SSGSEA immune cells. Interestingly, the risk model and immune risk model are both independent prognostic risk factors. Therefore, a nomogram combined risk score, immune risk score with clinical information which is meaningful in univariate and multivariate Cox regression analysis is established to predict the overall survival (OS) of COAD patients. In general, the signature consisted of 15 pyroptosis-related IncRNAs and was proved to be associated with the immune landscape of COAD patients.

Keywords: pyroptosis, IncRNA, colon adenocarcinoma (COAD), immune microenvironment, prognosis

\section{INTRODUCTION}

Colorectal cancer (CRC) is one of the most frequent malignancies and the third leading cause of cancer-related death globally. Based on the pathologic classification, colon adenocarcinoma (COAD) is the most common subtype of colon cancer (Sui et al., 2020; Yu et al., 2020). In recent years, the incidence of $\mathrm{COAD}$ has been rising among adolescents. Currently, the 5-years overall survival (OS) rate of COAD patients without distant metastasis has a good prognosis, but that of patients with metastases is less than 15\% (Almatroudi, 2020; Gan et al., 2020). Although the AJCC TNM staging system as the prognostic indicator of COAD patients is continually updated, a significantly different prognosis still exists in patients with the same clinicopathologic characteristics (Lino-Silva et al., 


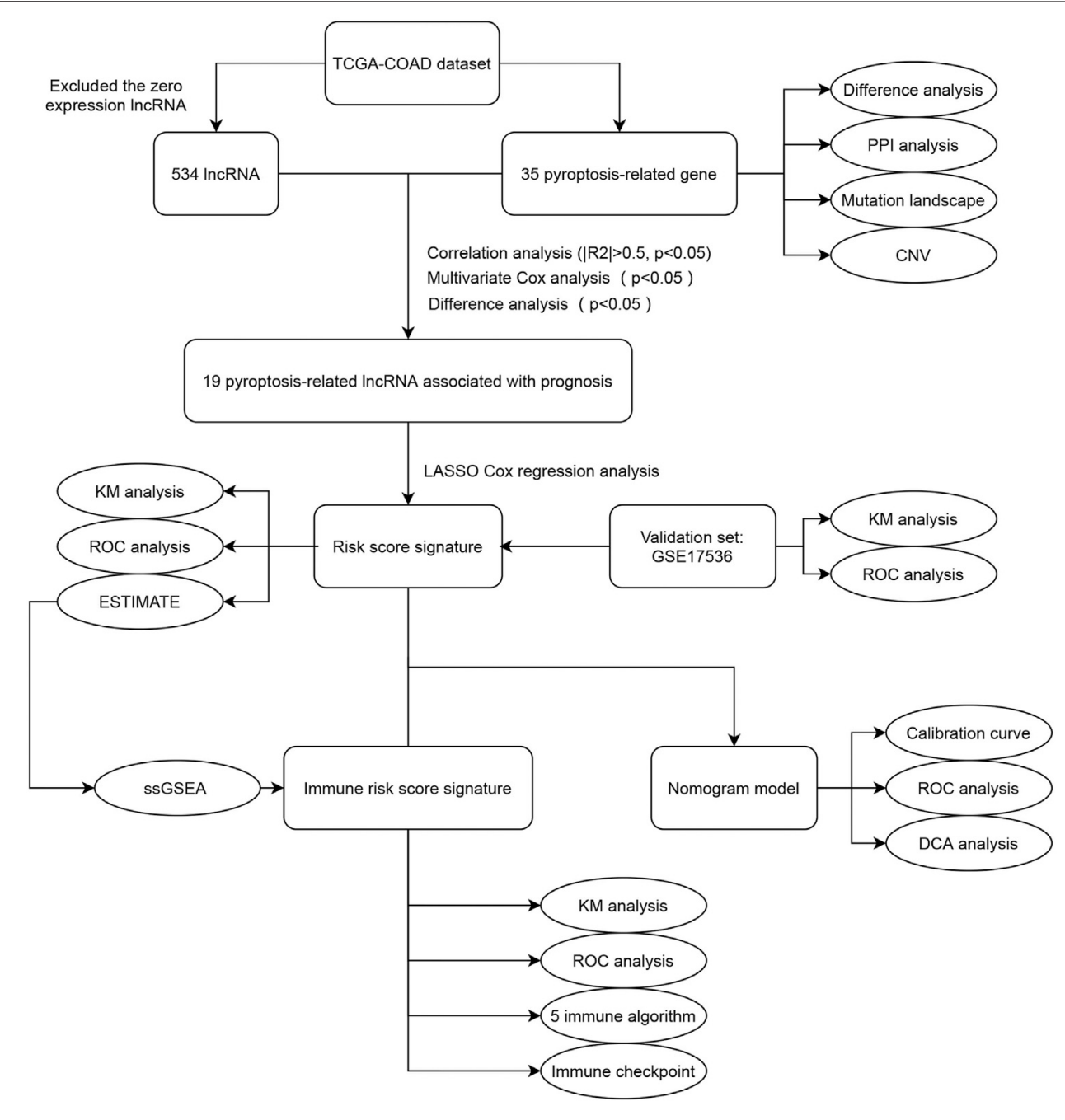

FIGURE 1 | The flowchart of this study. TCGA, The Cancer Genome Atlas; COAD, colon adenocarcinoma; PPI, protein-protein interaction; CNV, copy number variation; KM, Kaplan-Meier; ROC, receiver operating characteristic; ESTIMATE, Estimation of STromal and Immune cells in MAlignant Tumor tissues using Expression data; ssGSEA, single sample Gene Set Enrichment Analysis; DCA, decision curve analysis.

2020; Ren et al., 2020). Therefore, identifying specific and sensitive prognostic biomarkers is imperative to predict the outcomes of COAD patients.

Pyroptosis is a newly-found programmed cell death (PCD) dependent on inflammasome, characterized by pore formation in the plasma membrane, causing cell swelling and rupture, as well as the escape of proinflammatory contents (Kolbrink et al., 2020). Pyroptosis is a double-edged sword, playing a key role in resisting bacterial and viral infections. Meanwhile, it is directly associated with tumor development ( $\mathrm{Li}$ et al., 2020; Shi et al., 2020). Additionally, pyroptosis is related to the antitumor response by cross-linking between immunity and the cancer microenvironment (Xiang et al., 2020). Hence, it gives us a novel direction for the therapeutic targets of tumors. In recent years, some research has revealed the potential linkages between the pyroptosis-related pathways and COAD (Gong et al., 2021). However, the biological mechanism for the pyroptosis influence on tumor progression of COAD is still unclear.
LncRNA (long non-coding RNA) refers to a type of RNA that is more than $200 \mathrm{nt}$ in length and does not encode proteins but mediates gene expression at multiple levels (Lin et al., 2018). Numerous studies have identified that lncRNA is indispensable in tumorigenesis and development and even alters the tumor properties, leading to tumor microenvironment differences (Mercer et al., 2009; Qiu et al., 2021). Furthermore, the underlying mechanism between pyroptosis and lncRNAs remains to be deciphered, considering few studies elucidate the role of pyroptosis-related lncRNAs in predicting COAD prognosis and the association between those lncRNAs and the immune microenvironment. Thus, it is feasible to construct a risk model composed of pyroptosis-related lncRNAs, which can help us to precisely predict the prognosis of COAD patients and develop immunotherapies in the future.

In this study, we systematically explored the relationship between pyroptosis-related lncRNAs and probed into clinicopathological information and the tumor immune 
TABLE 1 | Characteristics of TCGA-COAD dataset.

\begin{tabular}{|c|c|c|c|c|c|}
\hline Characteristics & Tumor $(\mathrm{N}=429)$ & Normal $(\mathbf{N}=37)$ & Total $(\mathrm{N}=466)$ & $p$ Value & FDR \\
\hline Gender & & & & 0.99 & 1 \\
\hline Male & $227(48.71 \%)$ & 19 (4.08\%) & $246(52.79 \%)$ & & \\
\hline \multicolumn{6}{|l|}{ Age } \\
\hline Mean \pm SD & $66.70 \pm 12.77$ & $69.70 \pm 13.46$ & $66.94 \pm 12.84$ & & \\
\hline Disease type & & & & 0.11 & 0.66 \\
\hline Adenocarcinoma & 372 (79.83\%) & $28(6.01 \%)$ & 400 (85.84\%) & & \\
\hline Mucinous adenocarcinoma & $57(12.23 \%)$ & $9(1.93 \%)$ & $66(14.16 \%)$ & & \\
\hline Pathologic stage & & & & 0.18 & 0.88 \\
\hline Stage I & $74(15.88 \%)$ & $4(0.86 \%)$ & $78(16.74 \%)$ & & \\
\hline T stage & & & & 0.48 & 1 \\
\hline $\mathrm{T} 1$ & $9(1.93 \%)$ & $0(0.0 \mathrm{e}+0 \%)$ & $9(1.93 \%)$ & & \\
\hline T2 & 75 (16.09\%) & $4(0.86 \%)$ & $79(16.95 \%)$ & & \\
\hline Т3 & $297(63.73 \%)$ & $27(5.79 \%)$ & $324(69.53 \%)$ & & \\
\hline T4 & $48(10.30 \%)$ & $6(1.29 \%)$ & $54(11.59 \%)$ & & \\
\hline $\mathrm{N}$ stage & & & & 0.40 & 1 \\
\hline NO & 253 (54.29\%) & $26(5.58 \%)$ & 279 (59.87\%) & & \\
\hline $\mathrm{N} 1$ & $99(21.24 \%)$ & $6(1.29 \%)$ & $105(22.53 \%)$ & & \\
\hline N2 & $77(16.52 \%)$ & $5(1.07 \%)$ & $82(17.60 \%)$ & & \\
\hline M stage & & & & 0.62 & 1 \\
\hline MO & 367 (78.76\%) & 30 (6.44\%) & 397 (85.19\%) & & \\
\hline
\end{tabular}

$F D R$, false discovery rate; $S D$, standard deviation

landscape of COAD. We initially screened differentially expressed pyroptosis-related lncRNAs to build a prognostic risk model. Based on the median cut-off of risk score, we divided COAD patients into high-risk and low-risk groups. We also employed an external cohort to validate the prognostic predictive ability of the risk model. Then, the immune state of the two groups was assessed; accordingly, an immune risk model was constructed. To the end, we combined predictable clinicopathological features, risk score and immune risk score to construct an efficient nomogram to predict the survival rate of COAD patients. The detailed flowchart is shown in Figure 1. This study provides a new reference to predict the prognosis of COAD patients and identify personalized treatment strategies.

\section{MATERIALS AND METHODS}

\section{Datasets and Clinical Data}

The RNA-seq dataset of TCGA-COAD with matching clinical information was downloaded from the UCSC Xena browser (https://xena.ucsc.edu/). The gene annotation files (version GRCh38.99) were downloaded from the Ensembl genome browser (http://asia.ensembl.org/index.html). Patients without exact clinical information were excluded for the subsequent analysis. We got 429 COAD samples and 37 paired normal samples as the training cohort (Table 1). The GEO datasets (https://www.ncbi.nlm.nih.gov/geo/) with the accession number was GSE17536 (Smith et al., 2010), were used to verify the prognostic model. We discarded the cases with missing information in this analysis. The entire process was analyzed by $\mathrm{R}$ (version 4.0 .3 ).

\section{Identification of Pyroptosis-Related Genes}

Based on previous studies (Ye et al., 2021), we extracted a total of 35 pyroptosis-related genes for differentially expressed analysis; 26 of them were differentially expressed between normal and tumor samples through the rank sum test. The significance differences were indicated as follows: $p<0.05$ as ${ }^{*}, p<0.01$ as ${ }^{* *}$, and $p<0.001$ as ${ }^{* * *}$. The gene circular map showed mutated pyroptosis-related genes. The t-SNE was used to distinguish normal and tumor samples; the correlation analysis was used to discover interrelation based on the expression of pyroptosisrelated genes. The protein-protein interaction (PPI) was analyzed with STRING (https://string-db.org/) by setting the minimum interaction sources as 0.400 (Szklarczyk et al., 2021). 


\section{Identification of Pyroptosis-Related LncRNAs}

We removed lncRNAs with zero expression after annotation and then acquired $534 \operatorname{lncRNAs}$ for subsequent correlation analysis. Then, pyroptosis-related lncRNAs satisfied the correlation coefficient $\left|R^{2}\right|>0.5$ and $p<0.05$ by Pearson correlation analysis. The prognostic values of pyroptosis-related lncRNAs were assessed by multivariate Cox analysis $(p<0.05)$, and difference analysis was further conducted $(p<0.05)$. Finally, 19 pyroptosis-related lncRNAs were obtained to construct a prognostic risk signature.

\section{Development and Validation of Prognostic Pyroptosis-Related LncRNAs Signature}

Lasso Cox regression analysis was used to build a prognostic model to avoid overfitting (Tibshirani, 1997). We got 15 pyroptosis-related lncRNAs to construct the model by setting the $\lambda$ parameter as 0.009 through the "glmnet" R package. Each lncRNAs with coefficients contributed differently to prognosis, and each patients' risk scores were calculated by the following formula: risk score $=\sum_{i=0}^{n}$ expr $i *$ coef $i$. We divided COAD patients into the lowrisk and high-risk groups by setting the median risk score as the cutoff point. To compare the OS difference between the low- and highrisk groups, we used the "survminer" R package. To investigate the predictive ability of the prognostic model over time, we employed the "TimeROC" R package to show the time-dependent ROC curve. In order to confirm the broad applicability of the prognostic model, we introduced a validation set: GSE17536. The predictive ability of the model was repeatedly validated by the methods mentioned above.

\section{Development and Validation of Immune Infiltration Signature}

ESTIMATE is an algorithm for calculating tumor purity and the percentage of infiltrating stromal/immune cells in tumor samples (Yoshihara et al., 2013). After comparing the results of ESTIMATE between the low- and high-risk groups, we used ssGSEA (singlesample gene set enrichment analysis) to evaluate the infiltration of 28 immune cells. Also, using Lasso Cox regression analysis, an immune prognostic model was built by infiltrating scores of three immune cells. The Kaplan-Meier (K-M) survival analysis and timedependent ROC curve were performed to validate the predictive ability of the immune prognostic model. We employed more immune infiltration algorithms (CIBERSOFT, EPIC, IPS, MCP counter and TIMER) to assess the difference of immune microenvironment between the two immune-risk groups (Becht et al., 2016; Li et al., 2016; Charoentong et al., 2017; Racle et al., 2017; Newman et al., 2019). Immune checkpoint blockade (ICB) response was predicted by ImmuCellAI (http://bioinfo.life.hust. edu.cn/ImmuCellAI\#!/) to check the response of ICB in tumor samples (Miao et al., 2020).

\section{Construction of Nomogram}

The nomogram was based on the multivariate regression analysis of clinicopathological information, risk score and immune risk score. We used the "rms" R package to build the nomogram to predict the 1-, 3- and 5-years survival rates of COAD patients. The ROC curve and the calibration curve were utilized to estimate the suitability of clinical use. The alluvial plot was used to describe the shunt of each patient with different clinicopathological features. The DCA (decision curve analysis) was used to evaluate the clinical utility of the nomogram and help clinicians make decisions.

\section{Statistical Analysis}

$\mathrm{R}$ version 4.0.3 and various $\mathrm{R}$ packages were used to perform all statistical analyses. The chi-squared test was used to compare the constituent radio of two subgroups. The "Rtsne" $\mathrm{R}$ package was used to analyze $t$-SNE. The Pearson correlation analysis was used to evaluate the correlation between two variables. Univariate and multivariate Cox regression analysis was run by the "survival" $\mathrm{R}$ package. Hazard ratio $(\mathrm{HR})>1$ was considered as a risk factor, while HR > 1 was considered as a protective factor. The "GSVA" $\mathrm{R}$ package was utilized to perform the ssGSEA. The distribution of immune cell infiltration and immune check-point between the two groups was analyzed by the nonparametric test. The "rmda" $\mathrm{R}$ package was used to create the DCA curve.

\section{RESULTS}

\section{Survey of Different Expression and Genetic Alterations of Pyroptosis-Related Genes in COAD}

To find differently expressed pyroptosis-related genes in COAD, expression levels of 35 pyroptosis-related genes were compared between 429 tumor samples and 37 paired normal samples, identifying 26 genes representing significantly different expression $(p<0.05)$. Among them, 11 genes were upregulated, while the other 15 genes were downregulated in tumor samples (Figure 2A). Based on 26 differently expressed pyroptosis-related genes, t-SNE visualized the distribution between tumor and normal samples (Figure 2B). Also, correlation analysis (Figure 2C) and PPI network (Figure 2D) were performed to show the complex interactive relationship among the 26 pyroptosis-related genes. As shown in the histogram of interaction, IL-18 and IL-1 $\beta$ had the largest number of interactions (Figure 2E). At the level of genetic mutations, about 111 of 399 (approximately 27.82\%) tumor samples represented mutations of pyroptosis-related genes, and NLRP7 accounted for the most (Figure 2F). Then, we mapped these mutated pyroptosis-related genes on the chromosome (Figure 2G). The alterations of pyroptosisrelated genes might play an important role in $\mathrm{COAD}$ formation.

\section{Identification of Pyroptosis-Related LncRNAs With Prognostic Value}

Based on the TCGA-COAD RNA-seq dataset, 534 lncRNAs were included for further research by screening out lncRNAs with zero 

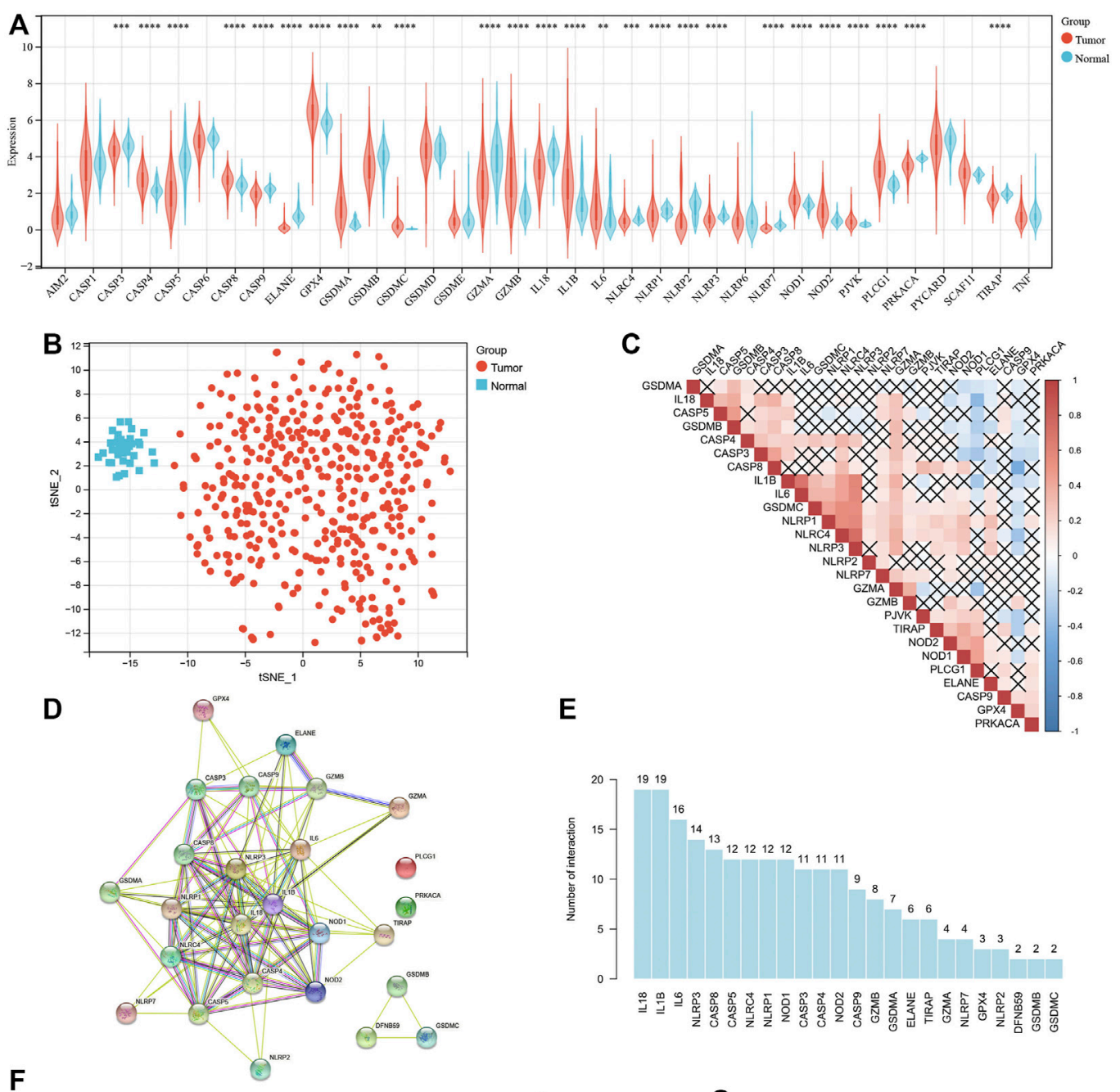

$E$
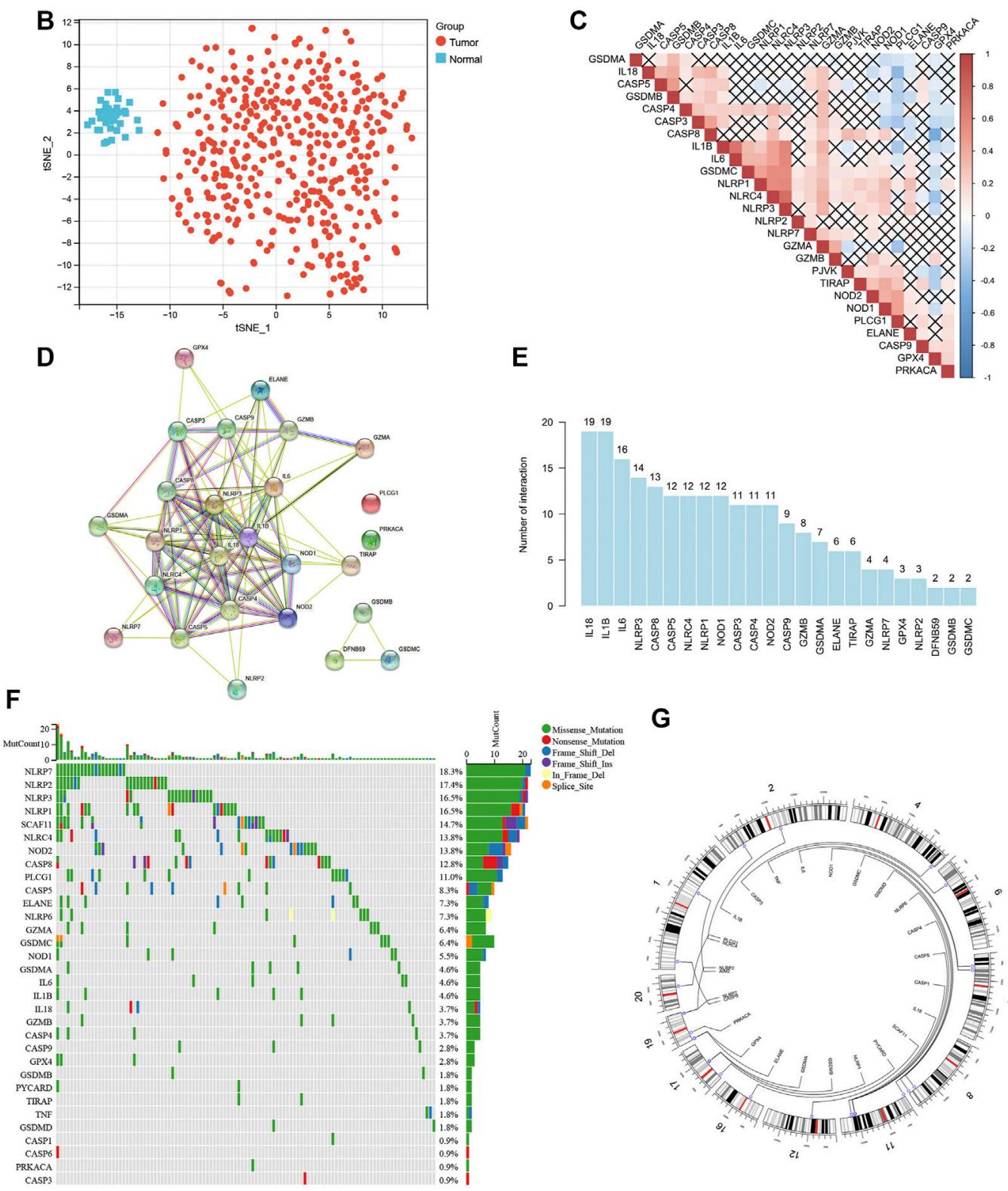

G

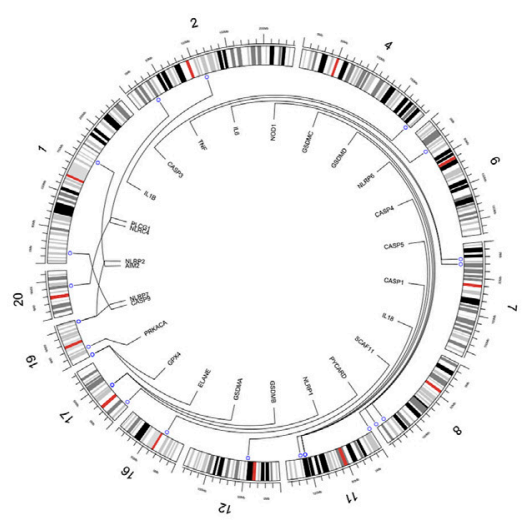

FIGURE 2 | Survey of different expression and genetic alterations of pyroptosis-related genes in COAD. (A) Boxplot of the expression levels of 35 pyroptosisrelated genes between tumor and normal samples. (B) $t$-SNE showing the distribution between tumor and normal samples. (C) Correlation among 16 differently expressed pyroptosis-related genes. (D) PPI network among 16 differently expressed pyroptosis-related genes. (E) Histogram of interaction among 16 differently expressed pyroptosis-related genes. (F) Mutation landscape of 399 COAD patients. Waterfall plot showing the mutation information of each pyroptosis-related gene. (G) The location of each pyroptosis-related gene on the chromosome. ${ }^{\star} p<0.05 ;{ }^{* \star} p<0.01 ;{ }^{\star \star *} p<0.001 ;{ }^{* \star \star} p<0.0001$. 


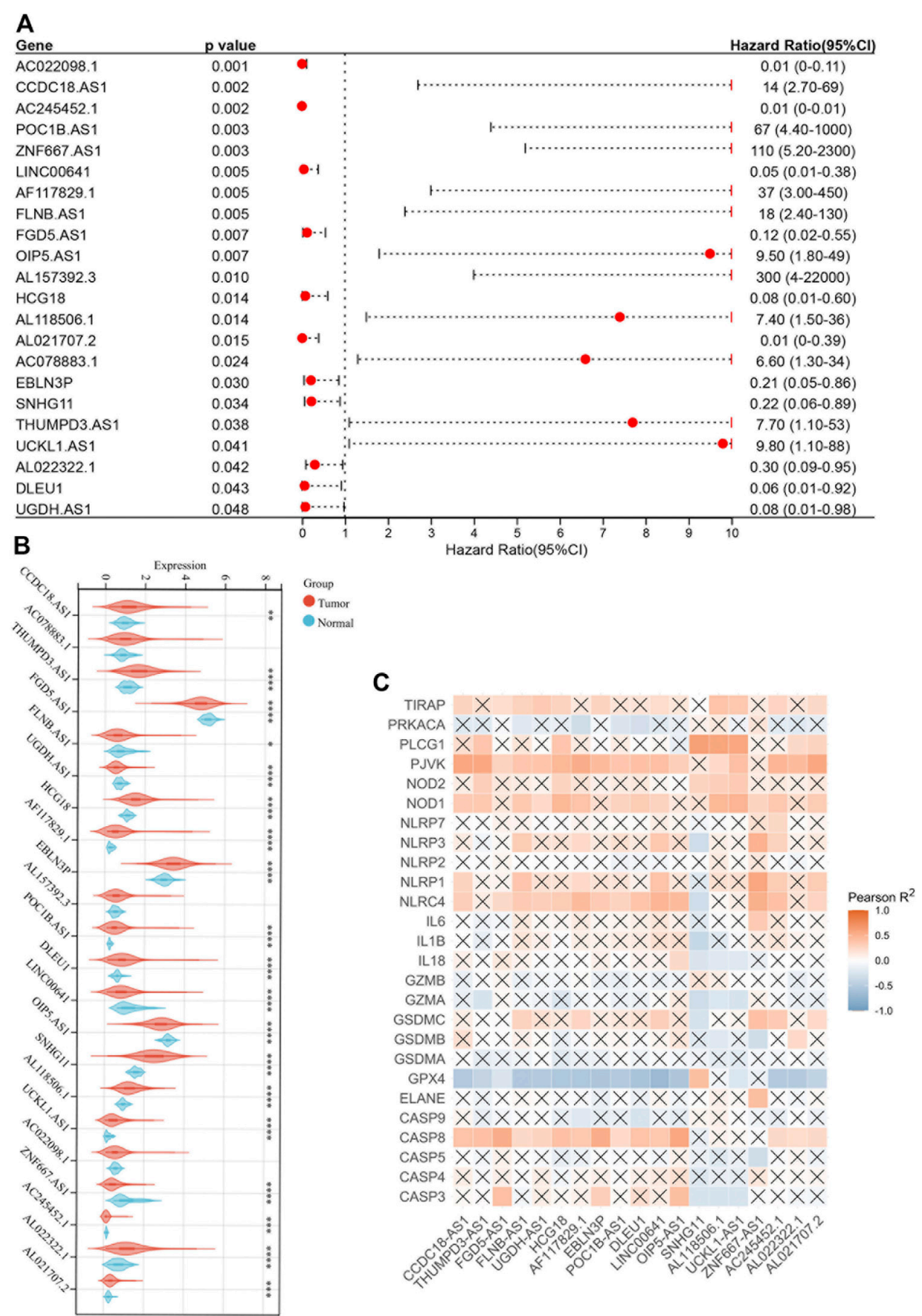

FIGURE 3 | Identification of pyroptosis-related IncRNAs with prognostic value. (A) Forest plot of 22 pyroptosis-related IncRNAs after multivariate Cox regression analysis $(p<0.05)$. (B) Boxplot of 22 pyroptosis-related IncRNAs. (C) Correlation between 22 pyroptosis-related IncRNAs and 26 pyroptosis-related genes. ${ }^{*} p<0.05$; ${ }^{\star \star} p<0.01 ;{ }^{* \star \star} p<0.001 ;{ }^{\star \star \star \star} p<0.0001$.

expression. Then, the correlation analysis between 26 pyroptosisrelated genes and $534 \operatorname{lncRNAs}$ was constructed under the specific conditions that Pearson correlation coefficient $\left|R^{2}\right|>$ 0.5 and $p<0.05$. After taking the 118 pyroptosis-related lncRNAs into multivariate Cox analysis $(p<0.05)$ and difference analysis $(p<0.05)$, we identified that 19 of them were associated with prognosis (all the results of multivariate Cox analysis were shown in Supplementary Material).

The forest plot showed 22 pyroptosis-related lncRNAs with prognostic significance after multivariate Cox analysis (Figure 3A). The correlation heatmap was plotted according to the difference analysis results (Figure 3B). Moreover, GPX4 

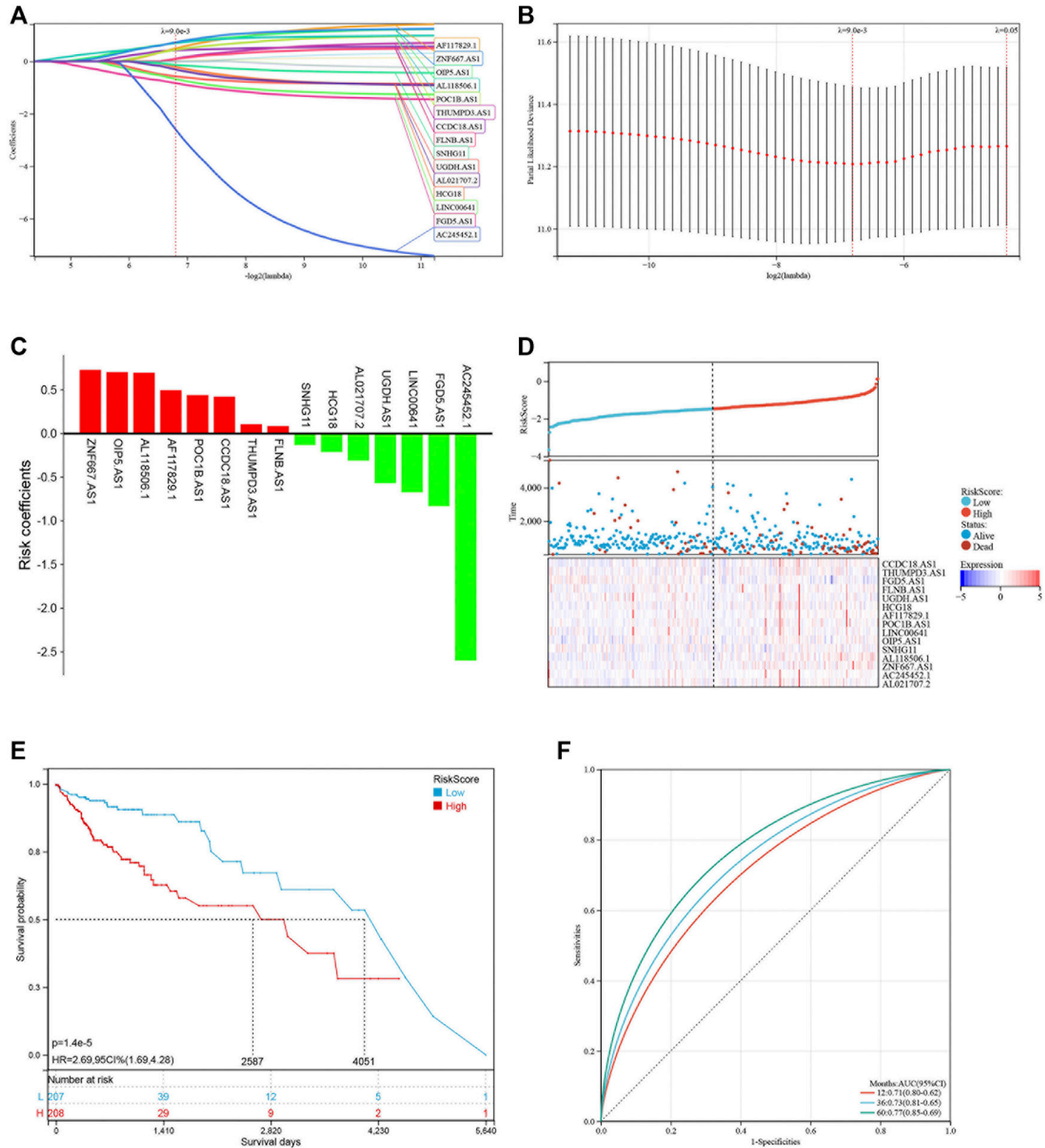

FIGURE 4 | Construction of the risk model based on pyroptosis-related IncRNAs. (A,B) LASSO regression of 16 pyroptosis-related IncRNAs. (C) LASSO coefficients of 16 pyroptosis-related IncRNAs. (D) Distribution of risk score and survival status of COAD patients. Heatmap showing the expression levels of 16 pyroptosis-related IncRNAs. (E) Kaplan-Meier curve showing the survival difference between low-risk and high-risk groups. (F) ROC curve of the risk score in predicting 1-, 3-, and 5-years OS.

was negatively correlated with most pyroptosis-related lncRNAs, while PJVK, NOD1, NLRC4 and CASP8 were positively correlated (Figure 3C).

\section{Construction and Validation of a Risk Model Based on Pyroptosis-Related LncRNAs}

Based on the 19 pyroptosis-related lncRNAs identified above, a risk model was created by Lasso Cox regreesion analysis to calculate the risk score of each patient (Figures 4A,B). By setting the compression parameter $\lambda=0.009,15 \operatorname{lncRNAs}$ were enrolled to build the risk model with each coefficient (Figure 4C). The risk score $=(\mathrm{ZNF} 667-\mathrm{AS} 1) \times(0.728,496)$

$+(\mathrm{OIP} 5-\mathrm{AS} 1) \times(0.704,723)+(\mathrm{AL} 118506.1) \times(0.695,672)$

$+(\mathrm{AF} 117829.1) \times(0.495,352)+(\mathrm{POC} 1 \mathrm{~B}-\mathrm{AS} 1) \times(0.441,168)$

$+($ CCDC18-AS1 $) \times(0.422,851)+($ THUMPD3-AS1 $)$

$\times(0.105,734)+($ FLNB-AS1 $) \times(0.086465)+($ SNHG11 $)$

$\times(-0.132,084)+($ HCG18) $\times(-0.211,577)+($ AL021707.2) 

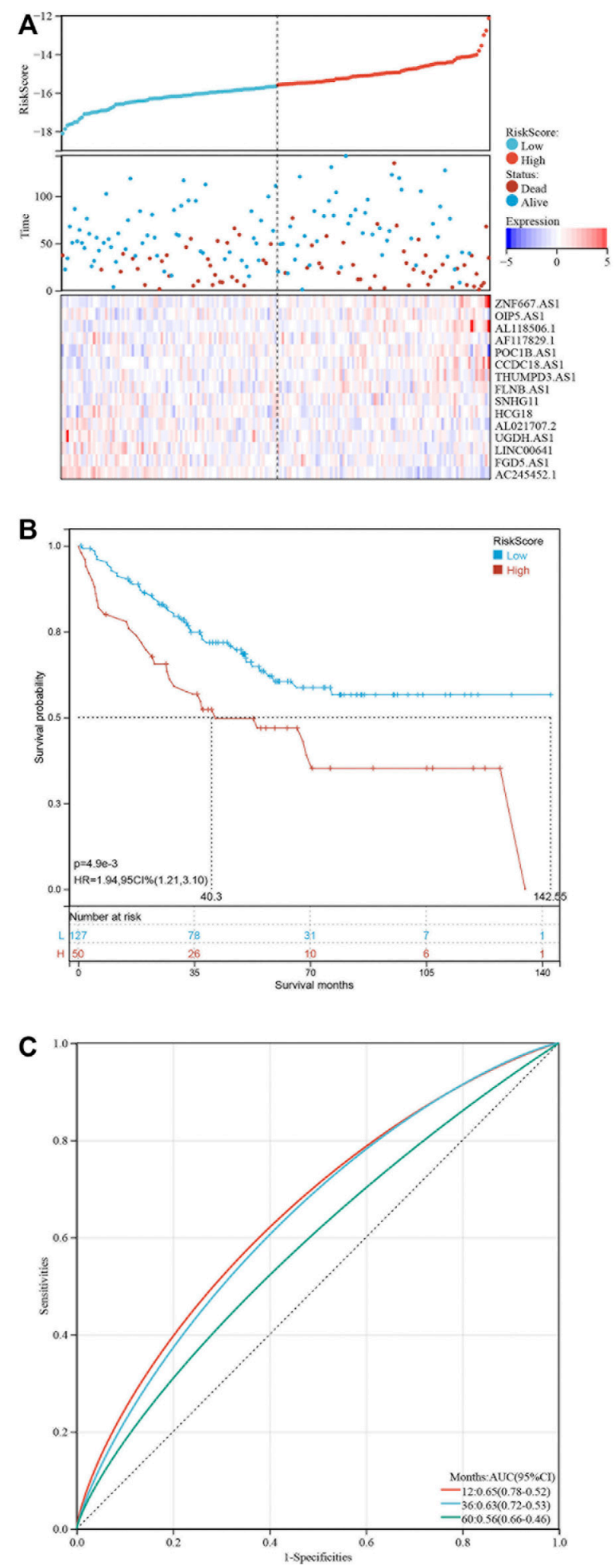

FIGURE 5 | Validation of the risk model at GSE17536. (A) Distribution of risk score and survival status of COAD patients. Heatmap showing the expression levels of 16 pyroptosis-related IncRNAs. (B) Kaplan-Meier curve showing the survival difference between low-risk and high-risk groups. (C) ROC curves of the risk score in predicting 1-, 3-, and 5years OS. $\times(-0.310,627)+($ UGDH-AS1 $) \times(-0.569,069)+($ LINC00641 $)$ $\times(-0.674,802)+($ FGD5-AS1 $) \times(-0.831,751)+($ AC245452.1 $)$ $\times(-2.598,539)$. Subsequently, we divided 429 COAD patients into high-risk and low-risk groups by setting the median risk score as the cut-off point (Figure 4D). As the risk score rose, the death numbers increased, and the survival time decreased. As shown by the KaplanMeier curve, COAD patients with high-risk scores had a poorer prognosis than those with low-risk scores (Figure 4E). Furthermore, the ROC curve demonstrated the predictive ability of the risk score in terms of prognosis; the areas under the curves (AUC) of 1,3 and 5 years were $0.71,0.73$ and 0.77 , respectively (Figure 4 F). For validation in external datasets, we employed GSE17536 as the validation set and repeated the above analysis (Figure 5A). The same results were obtained that patients with low-risk scores had a better prognosis (Figure 5B). The risk score had a good prognostic predictive ability with the AUC of $0.65,0.63$ and 0.56 in 1,3 and 5 years (Figure $5 \mathrm{C}$ ).

\section{Construction of an Immune Risk Model Based on Immune Cell Infiltration}

Emerging studies have pointed that immune-associated signaling pathways have important roles in cancer pathogenesis, including COAD (Xu et al., 2020; Zaiachuk et al., 2021). ESTIMATE was utilized to analyze the tumor immune microenvironment and score in each COAD sample. Interestingly, stromal score $(p<$ $0.001)$, ESTIMATE score $(p<0.01)$ and immune score $(p=0.10)$ were higher in the high-risk group than in the low-risk group, while tumor purity $(p<0.01)$ represented contrary results (Figure 6A). To better dissect the immune microenvironment, we utilized the ssGSEA algorithm to derive the infiltration degree of 28 immune cells in tumors. Lasso Cox regression analysis was performed again, and three immune cells were selected with the parameter $\lambda=0.03$ (Figure 6B). The immune risk score $=$ $($ CD56dim natural killer cell $) \times(1.916,028)+($ T follicular helper cell $\times(1.049875)+($ type $17 \mathrm{~T}$ helper cell $) \times(-5.093948)$ (Figure 6C). Based on the median immune risk score, COAD patients were divided into the high-immune-risk and lowimmune-risk groups. Likewise, a high immune risk score indicated a poor prognosis (Figure 6D). The prognostic predictive ability of the immune risk score was measured by the AUC to be $0.57,0.64$, and 0.60 in 1,3 and 5 years, respectively (Figure 6E). From the linear analysis, we found that the immune risk score was positively related to the risk score $(\mathrm{r}=0.27$ and $p<0.001)$ (Figure 6F). More immune algorithms were involved in demonstrating the immune microenvironment in multiple dimensions (Figure 7A). We further analyzed the expression levels of immune checkpoints between the two groups, and most of them were upregulated in the high-immune-risk group (Figure 7B). More patients in the high-immune-risk group (36\%) responded to ICB than those in the low-immune-risk group (29\%) (chi-squared test, $p=0.1395$ ) (Figure 7C).

\section{Development of a Nomogram Incorporated With Clinicopathological Information}

To better expend the clinical application of the signature, a nomogram integrating with clinicopathological information 
A
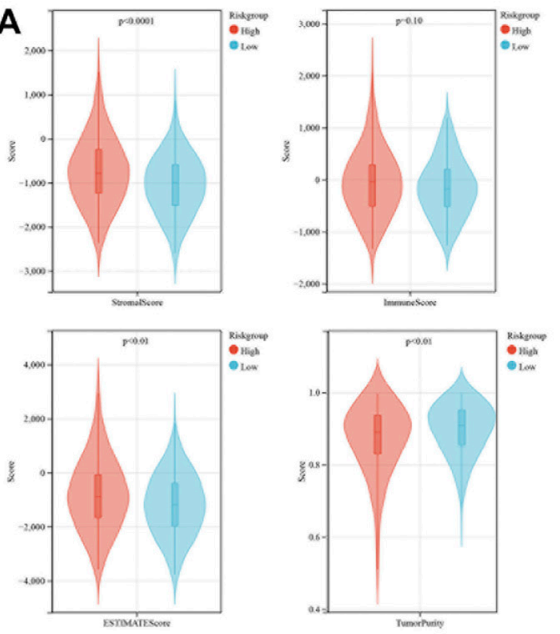

C

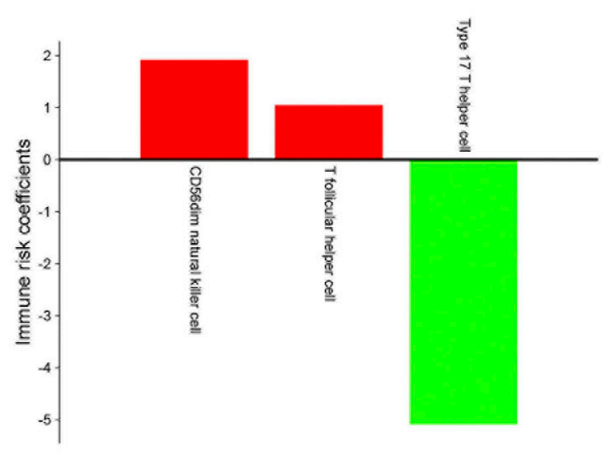

$E$

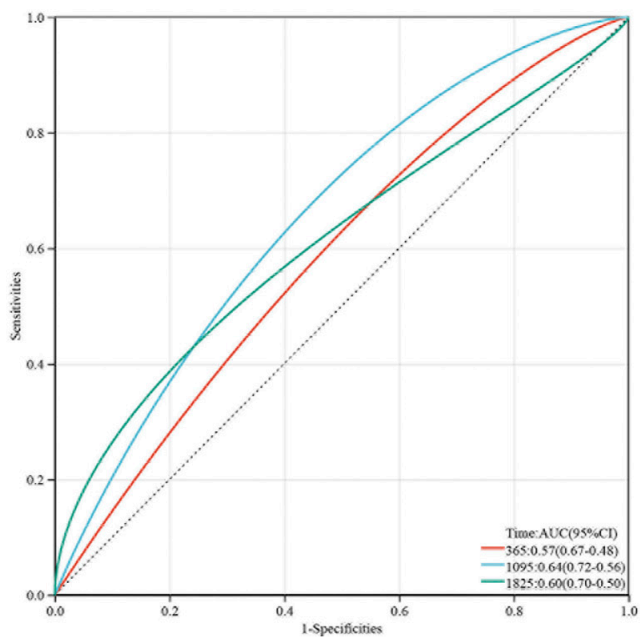

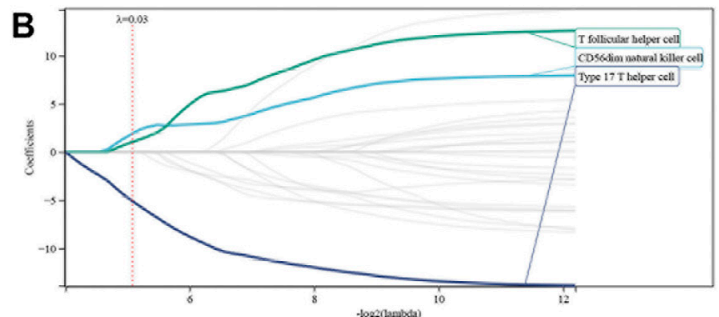

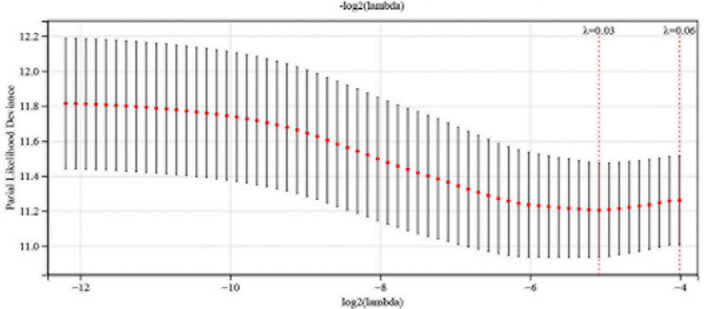

\section{D}

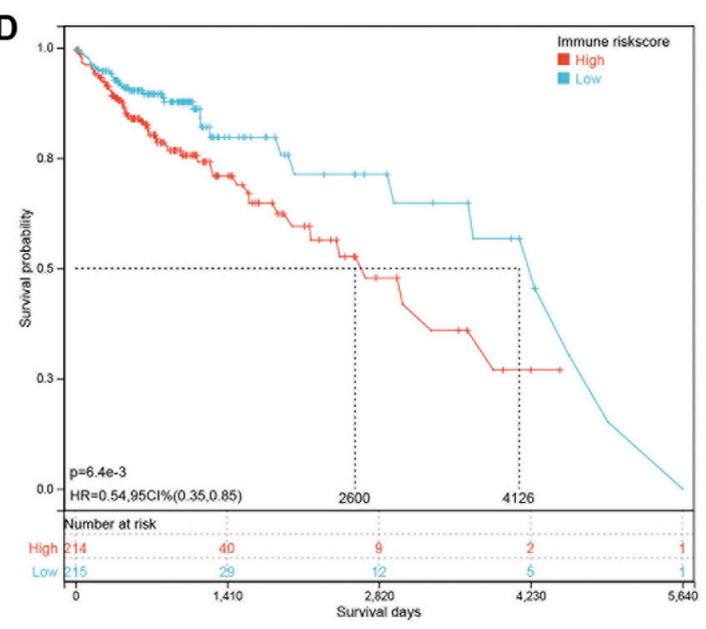

$\mathbf{F}$

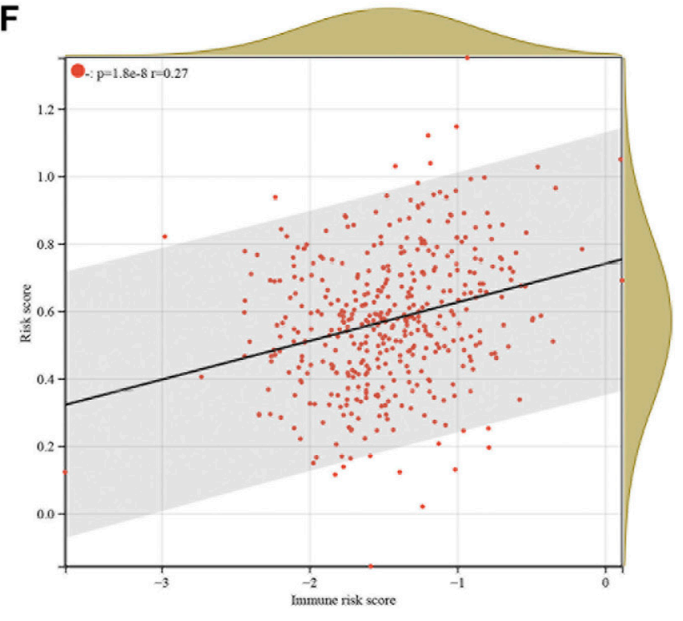

FIGURE 6 | Construction of an immune risk model based on immune cell infiltration. (A) Comparison of immune score, stromal score, ESTIMATE score and tumor purity between low-risk and high-risk groups. (B) LASSO coefficients of three immune cells. (C) LASSO coefficients of three immune cells. (D) Kaplan-Meier curve showing the survival difference between low-immune-risk and high-immune-risk groups. (E) ROC curves of the immune risk score in predicting 1-, 3-, and 5-years OS. (F) Linear relationship of risk score and immune risk score. 

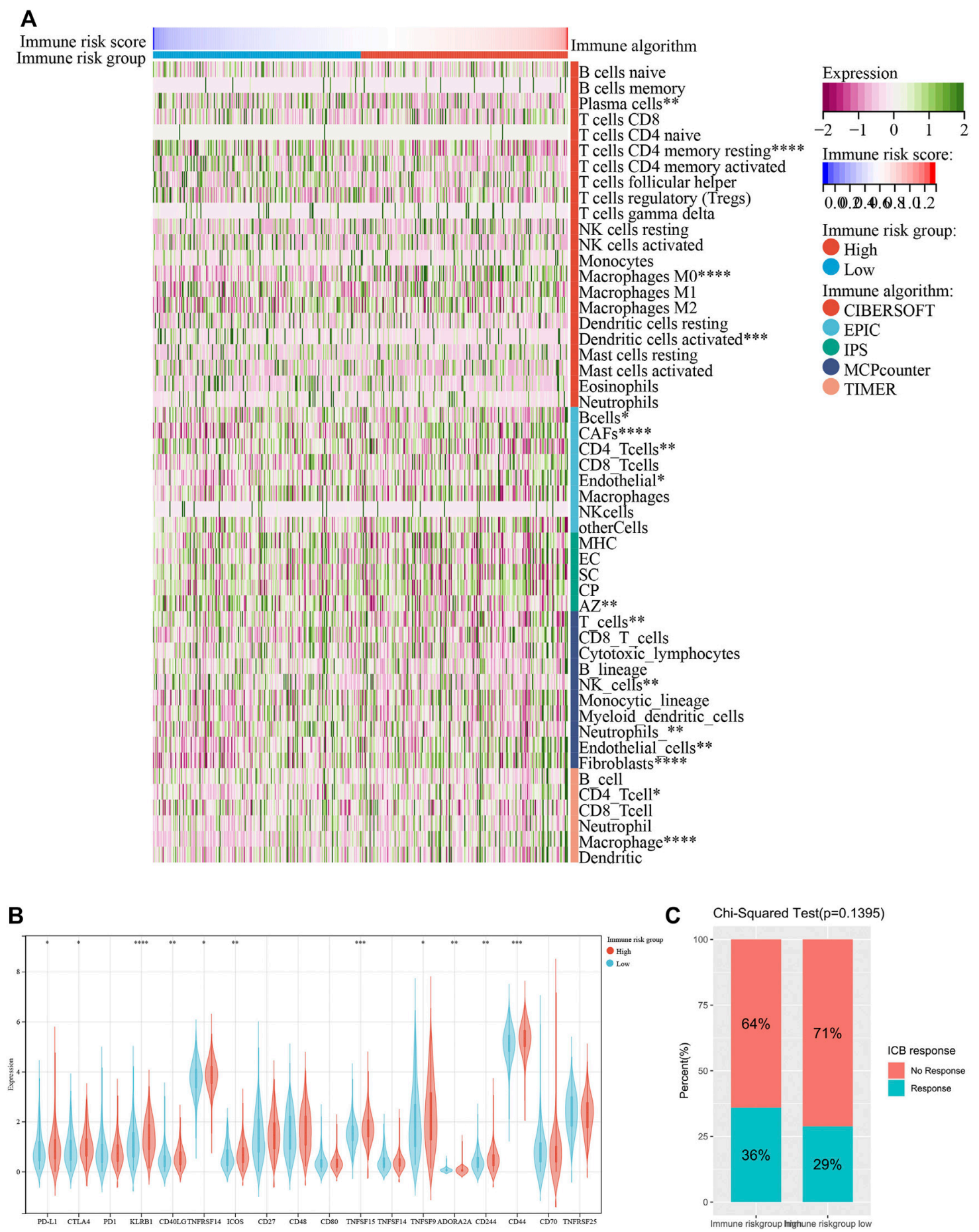

FIGURE 7 | Other immune algorithms employed in comparison between low-immune-risk and high-immune-risk groups. (A) Heatmap showing the infiltration levels of immune cells under CIBERSOF,, EPIC, IPS, MCP counter and TIMER algorithms. (B) Boxplot showing the expression levels of immune checkpoint between low-immune-risk and high-immune-risk groups. (C) Result of Chi-squared test of ICB response between low-immune-risk and high-immune-risk groups. ICB, immune checkpoint blockade. ${ }^{\star} p<0.05 ;{ }^{* \star} p<0.01 ;{ }^{* \star *} p<0.001 ;{ }^{\star \star \star \star} p<0.0001$.

was built. In the multivariate Cox analysis, we found that $\mathrm{N}$ stage, $\mathrm{M}$ stage, AJCC stage, risk score and immune risk score were significantly associated with prognosis, consistent with the result of univariate Cox analysis (Table 2). During the construction of the nomogram, the AJCC stage was the factor of overfitting, thus was excluded. By adding the fraction of 
TABLE 2 | Univariate and multivariate Cox regression analysis in TCGA-COAD dataset.

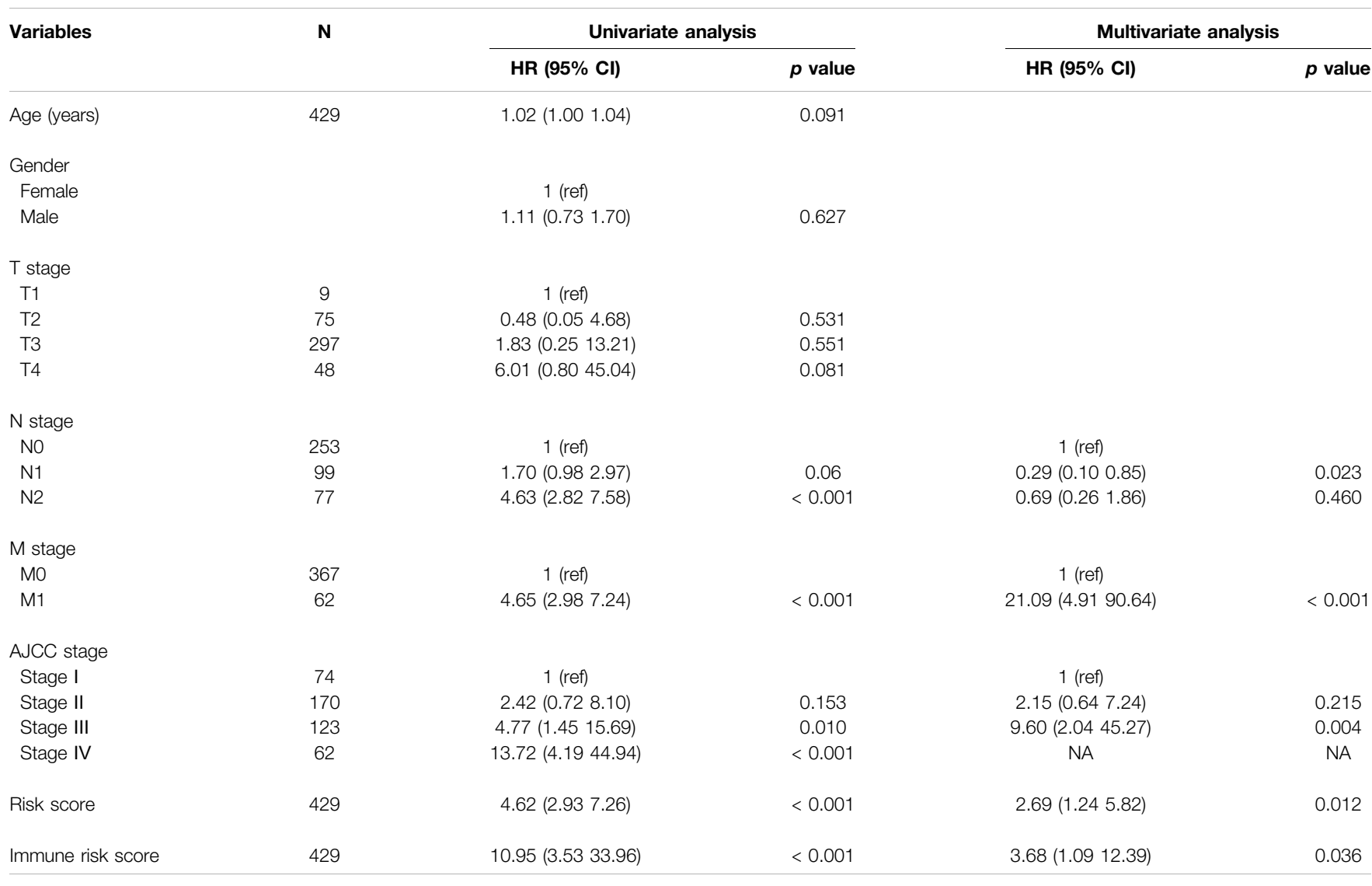

$\mathrm{HR}$, hazard ratio; $\mathrm{Cl}$, confidence interval; AJCC, American Joint Committee on Cancer

each prognostic characteristic, each patient had a total point representing the survival rate of 1, 3 and 5 years (Figure 8A). Higher points were associated with a worse prognosis. The alluvial plot visualized the outcome of each patient (Figure 8B). The calibration curve showed that the OS predictive probability of nomogram in 1, 3 and 5 years was consistent with that of the actual observed OS (Figure 8C). Meanwhile, the nomogram represented convincing sensitivity and specificity in predicting clinical outcomes, exhibited by the ROC curve with the AUC of 0.77, 0.81, and 0.79 in 1, 3, and 5 years, respectively (Figure $\mathbf{8 D}$ ). The DCA curve was used to solve the clinical utility problem of the nomogram. Compared with the traditional clinicopathological features, the nomogram had the optimum net benefit (NB) (Figure 8E).

\section{DISCUSSION}

Our study indicated that the prognostic signature based on the pyroptosis-related lncRNAs was a mighty predictive method for COAD patients. Meanwhile, the relation between risk score and immune microenvironment showed a potential contributing factor to poor prognosis. Hence, after integrating traditional clinicopathological information, risk score with the immune risk score, we constructed the unique prognostic evaluation system. The nomogram established a connection between the gene level and clinical prognosis. Factors affecting the prognosis of COAD patients were considered from multiple dimensions, forming a solid foundation for our clinical treatment.

Pyroptosis is a unique programmed cell death modality distinct from apoptosis. With research progressing, pyroptosis has been implicated in the activation of caspase-1, $-4,-5,-11$ and the maturation and release inflammatory cytokines IL- $1 \beta$ and IL18 (Wang et al., 2019). As reported in numerous studies, pyroptosis is present in macrophages infected by HIV, dengue virus, adenovirus, etc. (Chen et al., 2012). In addition, pyroptosis has been reported to be involved in atherosclerosis and gouty arthritis. The present study identified the presence of inflammasomes in tumor cells that direct caspase-1 for pyroptosis, which may be an important node in the association of tumorigenesis and development. For example, the inflammasome NLRP3 is widespread in CRC, lung adenocarcinoma, etc. (Zhang et al., 2018). Although pyroptosis can inhibit tumor development, it is pro-inflammatory, creating a microenvironment suitable for tumor growth and thus promoting tumor growth (Xia et al., 2019). Therefore, we focused our efforts on exploring the interactive patterns of pyroptosis in COAD and developed a prognostic predictive signature. 


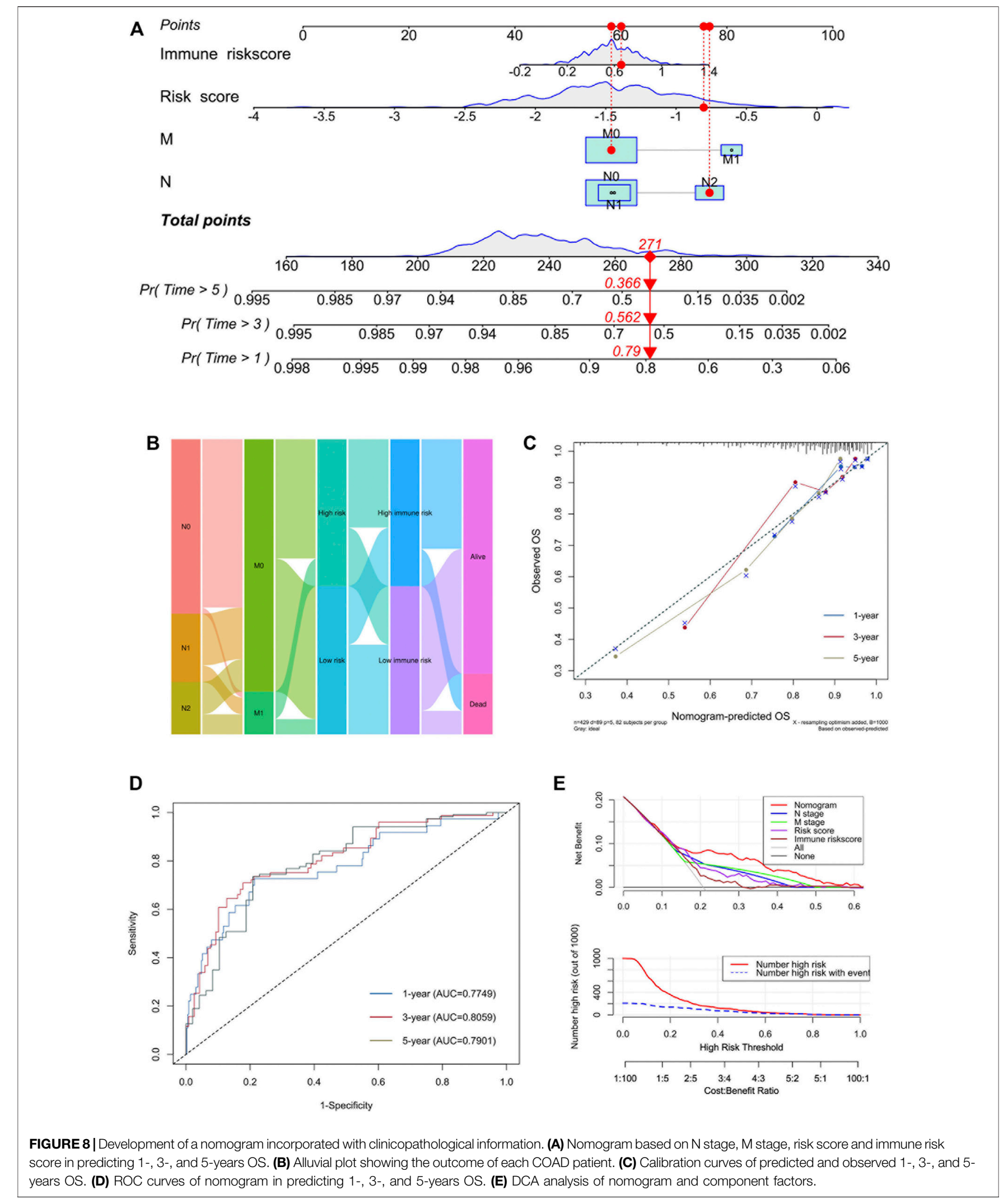


We identified 15 pyroptosis-related lncRNAs in this study, and some of them were already reported to exist in COAD or other cancers. Among them, ZNF667-AS1, OIP5-AS1, AL118506.1, AF117829.1, POC1B-AS1, CCDC18-AS1, THUMPD3-AS1 and FLNB-AS1 were risk-related lncRNAs, while SNHG11, HCG18, AL021707.2, UGDH-AS1, LINC00641, FGD5-AS1 and AC245452.1 were protective lncRNAs. ZNF667-AS1 (lncRNA mortal obligate RNA transcript) plays an important role in gynecological cancers and might be a potential diagnostic marker (Di Fiore et al., 2021). OIP5-AS1 (OIP5 antisense transcript 1), highly expressed in the nervous system and involved in the regulation of the cell cycle, is closely associated with neoplastic transformation (Ghafouri-Fard et al., 2021). In bone metastatic melanoma, AL118506.1 (antisense to abhydrolase domain containing 16B) participates in the ceRNA network of has-miR-27b-3p/AL118506.1/THBS2, which has great utility in predicting survival and metastasis of melanoma (Huang et al., 2019). In severe aplastic anemia (SAA) patients, the decreased expression of AF117829.1 upregulates the activity of $\mathrm{CD}^{+} \mathrm{T}$ lymphocytes, which means lncRNA may be associated with the regulation of the immune microenvironment (Li et al., 2021). POC1B-AS1 is also a risk factor in the prognostic model of endometrial carcinoma (Liu J. et al., 2021). In psoriasis, CCDC18-AS1, regarded as immune-related lncRNA, can be a potential diagnostic biomarker (Fan et al., 2021). THUMPD3-AS1 can regulate self-renewal by ceRNA network of miR-543 and ONECUT2 associated with non-small cell lung cancer ( $\mathrm{Hu}$ et al., 2019). The expression levels of FLNB-AS1 are positively correlated with the survival probability of breast cancer patients (Zhang et al., 2020). The SNHG11/miR-184/CDC25A ceRNA network is a new biomarker for the diagnosis, treatment and prognosis of gastric cancer (Zhao et al., 2021). A previous study has shown that HCG18 participates in vascular invasion of hepatocellular cancer by regulating immune cells (Zhang et al., 2021). Interestingly, AL021707.2, as a part of ferroptosisrelated lncRNAs signature of lung adenocarcinoma, can predict the prognosis of lung adenocarcinoma, which is the same as LINC00641 in glioma (Zheng J. et al., 2021; Zheng Z. et al., 2021). UGDH-AS1 has a prognostic value in esophageal cancer (Liu et al., 2020). The activation of FGD5-AS1 can promote the progression of cervical cancer by regulating BST2 to inhibit macrophage M1 polarization (Liu G. et al., 2021).

For a better assessment of the scientific nature of the model building, we compared our pyroptosis-related lncRNAs signature with that of other cancers. In lung adenocarcinoma, Song et al. constructed a pyroptosis-related lncRNAs signature for prognostic prediction (Song et al., 2021). Their idea of finding the pyroptosis-related lncRNAs to build the model was basically similar to ours. The clinical information was also combined to construct the nomogram, and the tumor microenvironment was analyzed. However, the value of the Pearson correlation coefficient was small, and there were no external datasets to validate their signature. Tang et al. built a pyroptosis-related lncRNAs signature for predicting the prognosis of clear cell renal cell carcinoma (ccRCC) (Tang et al., 2021). They performed a large enrichment analysis to reveal the roles of pyroptosis-related lncRNAs in ccRCC from their biological functions. Still, their study required additional confirmation in external datasets. Prior to our study, the association of pyroptosis with colon cancer was similarly explored. Wei et al. conducted a comprehensive bioinformatics analysis of the role of pyroptosis in the development of colon cancer (Wei et al., 2021). They clustered colon cancer samples according to pyroptosisrelated genes and obtained three pyroptosis-related subtypes. Then, weighted gene co-expression network analysis (WGCNA) showed that tumor microenvironment was significantly associated with pyroptosis-related subtypes. Finally, the genes in the module were combined with meaningful clinical features of the multivariate Cox analysis to predict the prognosis of colon cancer patients. These studies gave us much inspiration, on which we further incorporated pyroptosis-related lncRNAs for analysis. Thus, we constructed a risk model not only for pyroptosis-related lncRNAs but also for immune infiltrating cells. Deeper insights revealed the tight link between pyroptosis and colon cancer development as well as the tumor microenvironment.

Up to now, all the pyroptosis-related lncRNAs included in constructing the prognostic signature of COAD have been confirmed. Among them is no shortage of those about ferroptosis and apoptosis, which are other forms of programmed cell death. Furthermore, the interaction between these lncRNAs and the immune microenvironment is close and complicated. Therefore, we have reasons to believe that the lncRNAs prognostic signature associated with pyroptosis in COAD has its feasibility. Indubitably, there are some limitations in this study. The validation set from GEO just verified the prediction efficiency of the risk model based on the 15 pyroptosis-related lncRNAs, without conjunction with clinical information. Fundamental experiments of tumor cell lines and clinical samples are missing. Therefore, more experiments are needed to explore the molecular mechanism between the lncRNAs and pyroptosis in COAD.

\section{CONCLUSION}

To sum up, the pyroptosis-related lncRNAs signature exhibited its capability of predicting the clinical outcomes of COAD patients and participated in the immune microenvironment cellularity. We hope that our findings will provide a basis for the diagnosis and treatment of COAD in the future.

\section{DATA AVAILABILITY STATEMENT}

Publicly available datasets were analyzed in this study. This data can be found here: https://portal.gdc.cancer.gov/, https://www. ncbi.nlm.nih.gov/geo/. 


\section{AUTHOR CONTRIBUTIONS}

Acquisition of Data: CS Analysis and Interpretation of Data: CS Conception and Design: FX Data Curation: YY Development of Methodology: YY Writing the Manuscript: CS and YY.

\section{FUNDING}

This study was supported by the science and technology innovation Program of Hunan Province (No. 2021RC3029) and the National Natural Science Foundation of China (No. 81803035).

\section{REFERENCES}

Almatroudi, A. (2020). The Incidence Rate of Colorectal Cancer in Saudi Arabia: An Observational Descriptive Epidemiological Analysis. Int. J. Gen. Med. 13, 977-990. doi:10.2147/IJGM.S277272

Becht, E., Giraldo, N. A., Lacroix, L., Buttard, B., Elarouci, N., Petitprez, F., et al. (2016). Estimating the Population Abundance of Tissue-Infiltrating Immune and Stromal Cell Populations Using Gene Expression. Genome Biol. 17 (1), 218. doi:10.1186/s13059-016-1070-5

Charoentong, P., Finotello, F., Angelova, M., Mayer, C., Efremova, M., Rieder, D., et al. (2017). Pan-Cancer Immunogenomic Analyses Reveal GenotypeImmunophenotype Relationships and Predictors of Response to Checkpoint Blockade. Cel Rep. 18 (1), 248-262. doi:10.1016/j.celrep.2016.12.019

Chen, L. C., Wang, L. J., Tsang, N. M., Ojcius, D. M., Chen, C. C., Ouyang, C. N., et al. (2012). Tumour Inflammasome-Derived IL-1 $\beta$ Recruits Neutrophils and Improves Local Recurrence-Free Survival in EBV-Induced Nasopharyngeal Carcinoma. EMBO Mol. Med. 4 (12), 1276-1293. doi:10.1002/emmm.201201569

Di Fiore, R., Suleiman, S., Drago-Ferrante, R., Felix, A., O’Toole, S. A., O’Leary, J. J., et al. (2021). LncRNA MORT (ZNF667-AS1) in Cancer-Is There a Possible Role in Gynecological Malignancies? Int. J. Mol. Sci. 22 (15), 7829. doi:10.3390/ ijms22157829

Fan, F., Huang, Z., and Chen, Y. (2021). Integrated Analysis of Immune-Related Long Noncoding RNAs as Diagnostic Biomarkers in Psoriasis. PeerJ 9, e11018. doi:10.7717/peerj. 11018

Gan, G.-L., Liu, J., Chen, W.-J., Ye, Q.-Q., Xu, Y., Wu, H.-T., et al. (2020). The Diverse Roles of the Mucin Gene Cluster Located on Chromosome 11p15.5 in Colorectal Cancer. Front. Cel Dev. Biol. 8, 514. doi:10.3389/fcell.2020.00514

Ghafouri-Fard, S., Dashti, S., Farsi, M., Hussen, B. M., and Taheri, M. (2021). A Review on the Role of Oncogenic lncRNA OIP5-AS1 in Human Malignancies. Biomed. Pharmacother. 137, 111366. doi:10.1016/j.biopha.2021.111366

Gong, W., Liu, P., Zhao, F., Liu, J., Hong, Z., Ren, H., et al. (2021). STING-Mediated Syk Signaling Attenuates Tumorigenesis of Colitis-Associated Colorectal Cancer through Enhancing Intestinal Epithelium Pyroptosis. Inflamm. Bowel Dis. [Epub ahead of print], izab217. doi:10.1093/ibd/izab217

Hu, J., Chen, Y., Li, X., Miao, H., Li, R., Chen, D., et al. (2019). THUMPD3-AS1 Is Correlated with Non-Small Cell Lung Cancer and Regulates Self-Renewal through miR-543 and ONECUT2. Onco Targets Ther. 12, 9849-9860. doi:10.2147/OTT.S227995

Huang, R., Zeng, Z., Li, G., Song, D., Yan, P., Yin, H., et al. (2019). The Construction and Comprehensive Analysis of ceRNA Networks and TumorInfiltrating Immune Cells in Bone Metastatic Melanoma. Front. Genet. 10, 828. doi:10.3389/fgene.2019.00828

Kolbrink, B., Riebeling, T., Kunzendorf, U., and Krautwald, S. (2020). Plasma Membrane Pores Drive Inflammatory Cell Death. Front. Cel Dev. Biol. 8, 817. doi:10.3389/fcell.2020.00817

Li, B., Severson, E., Pignon, J.-C., Zhao, H., Li, T., Novak, J., et al. (2016). Comprehensive Analyses of Tumor Immunity: Implications for Cancer Immunotherapy. Genome Biol. 17 (1), 174. doi:10.1186/s13059-016-1028-7

Li, L.-L., Dai, B., Sun, Y.-H., and Zhang, T.-T. (2020). The Activation of IL-17 Signaling Pathway Promotes Pyroptosis in Pneumonia-Induced Sepsis. Ann. Transl Med. 8 (11), 674. doi:10.21037/atm-19-1739

\section{ACKNOWLEDGMENTS}

All authors thank TCGA and GEO databases for providing the worthy data and the Sangerbox tools which is a free online platform for data analysis.

\section{SUPPLEMENTARY MATERIAL}

The Supplementary Material for this article can be found online at: https://www.frontiersin.org/articles/10.3389/fcell.2021.811734/ full\#supplementary-material

Li, Y., Deng, L., Pan, X., Liu, C., Fu, R., and Qin, S. (2021). The Role of lncRNA AF117829.1 in the Immunological Pathogenesis of Severe Aplastic Anaemia. Oxidative Med. Cell Longevity 2021, 1-19. doi:10.1155/2021/5587921

Lin, Y.-T., Liu, W., He, Y., Wu, Y.-L., Chen, W.-N., Lin, X.-J., et al. (2018). Hepatitis B Virus X Protein Increases 8-Oxo-7,8-Dihydro-2'-Deoxyguanosine (8-Oxodg) Level via Repressing MTH1/MTH2 Expression in Hepatocytes. Cell Physiol Biochem 51 (1), 80-96. doi:10.1159/000495166

Lino-Silva, L. S., Xinaxtle, D. L., and Salcedo-Hernández, R. A. (2020). Tumor Deposits in Colorectal Cancer: The Need for a New "pN" Category. Ann. Transl Med. 8 (12), 733. doi:10.21037/atm.2020.03.175

Liu, H., Zhang, Q., Lou, Q., Zhang, X., Cui, Y., Wang, P., et al. (2020). Differential Analysis of IncRNA, miRNA and mRNA Expression Profiles and the Prognostic Value of IncRNA in Esophageal Cancer. Pathol. Oncol. Res. 26 (2), 1029-1039. doi:10.1007/s12253-019-00655-8

Liu, G., Du, X., Xiao, L., Zeng, Q., and Liu, Q. (2021a). Activation of FGD5-AS1 Promotes Progression of Cervical Cancer through Regulating BST2 to Inhibit Macrophage M1 Polarization. J. Immunol. Res. 2021, 5857214. doi:10.1155/ 2021/5857214

Liu, J., Mei, J., Wang, Y., Chen, X., Pan, J., Tong, L., et al. (2021b). Development of a Novel Immune-Related lncRNA Signature as a Prognostic Classifier for Endometrial Carcinoma. Int. J. Biol. Sci. 17 (2), 448-459. doi:10.7150/ijbs.51207

Mercer, T. R., Dinger, M. E., and Mattick, J. S. (2009). Long Non-Coding RNAs: Insights into Functions. Nat. Rev. Genet. 10 (3), 155-159. doi:10.1038/nrg2521

Miao, Y. R., Zhang, Q., Lei, Q., Luo, M., Xie, G. Y., Wang, H., et al. (2020). ImmuCellAI: A Unique Method for Comprehensive T-Cell Subsets Abundance Prediction and its Application in Cancer Immunotherapy. Adv. Sci. 7 (7), 1902880. doi:10.1002/advs.201902880

Newman, A. M., Steen, C. B., Liu, C. L., Gentles, A. J., Chaudhuri, A. A., Scherer, F., et al. (2019). Determining Cell Type Abundance and Expression from Bulk Tissues with Digital Cytometry. Nat. Biotechnol. 37 (7), 773-782. doi:10.1038/s41587-019-0114-2

Qiu, Y., Xu, M., and Huang, S. (2021). Long Noncoding RNAs: Emerging Regulators of normal and Malignant Hematopoiesis. Blood [Epub ahead of print]. doi:10.1182/blood.2021011992

Racle, J., de Jonge, K., Baumgaertner, P., Speiser, D. E., and Gfeller, D. (2017) Simultaneous Enumeration of Cancer and Immune Cell Types from Bulk Tumor Gene Expression Data. eLife 6, e26476. doi:10.7554/eLife.26476

Ren, X., Chen, B., Hong, Y., Liu, W., Jiang, Q., Yang, J., et al. (2020). The Challenges in Colorectal Cancer Management during COVID-19 Epidemic. Ann. Transl Med. 8 (7), 498. doi:10.21037/atm.2020.03.158

Shi, C., Wang, Q., Rao, Z., Shi, Y., Wei, S., Wang, H., et al. (2020). Diabetes Induces Hepatocyte Pyroptosis by Promoting Oxidative Stress-Mediated NLRP3 Inflammasome Activation during Liver Ischaemia and Reperfusion Injury. Ann. Transl Med. 8 (12), 739. doi:10.21037/atm-20-1839

Smith, J. J., Deane, N. G., Wu, F., Merchant, N. B., Zhang, B., Jiang, A., et al. (2010). Experimentally Derived Metastasis Gene Expression Profile Predicts Recurrence and Death in Patients with Colon Cancer. Gastroenterology 138 (3), 958-968. doi:10.1053/j.gastro.2009.11.005

Song, J., Sun, Y., Cao, H., Liu, Z., Xi, L., Dong, C., et al. (2021). A Novel Pyroptosis-Related lncRNA Signature for Prognostic Prediction in Patients with Lung Adenocarcinoma. Bioengineered 12 (1), 5932-5949. doi:10.1080/ 21655979.2021.1972078 
Sui, X., Chen, Y., Liu, B., Li, L., Huang, X., Wang, M., et al. (2020). The Relationship between KRAS Gene Mutation and Intestinal flora in Tumor Tissues of Colorectal Cancer Patients. Ann. Transl Med. 8 (17), 1085. doi:10.21037/ atm-20-5622

Szklarczyk, D., Gable, A. L., Nastou, K. C., Lyon, D., Kirsch, R., Pyysalo, S., et al. (2021). The STRING Database in 2021: Customizable Protein-Protein Networks, and Functional Characterization of User-Uploaded Gene/Measurement Sets. Nucleic Acids Res. 49 (D1), D605-D612. doi:10.1093/nar/gkaa1074

Tang, X., Zhang, A., Feng, Y., Su, Y., Wang, X., Jiang, F., et al. (2021). A Novel Pyroptosis-Related IncRNAs Signature for Predicting the Prognosis of Kidney Renal Clear Cell Carcinoma and its Associations with Immunity. J. Oncol. 2021, 1-15. doi:10.1155/2021/9997185

Tibshirani, R. (1997). The Lasso Method for Variable Selection in the Cox Model. Statist. Med. 16 (4), 385-395. doi:10.1002/(sici)10970258(19970228)16:4<385:aid-sim380>3.0.co;2-3

Wang, Y.-Y., Liu, X.-L., and Zhao, R. (2019). Induction of Pyroptosis and its Implications in Cancer Management. Front. Oncol. 9, 971. doi:10.3389/ fonc.2019.00971

Wei, R., Li, S., Yu, G., Guan, X., Liu, H., Quan, J., et al. (2021). Deciphering the Pyroptosis-Related Prognostic Signature and Immune Cell Infiltration Characteristics of Colon Cancer. Front. Genet. 12, 755384. doi:10.3389/ fgene.2021.755384

Xia, X., Wang, X., Cheng, Z., Qin, W., Lei, L., Jiang, J., et al. (2019). The Role of Pyroptosis in Cancer: Pro-Cancer or Pro-"Host"? Cell Death Dis 10 (9), 650. doi:10.1038/s41419-019-1883-8

Xiang, H., Zhu, F., Xu, Z., and Xiong, J. (2020). Role of Inflammasomes in Kidney Diseases via Both Canonical and Non-canonical Pathways. Front. Cel Dev. Biol. 8, 106. doi:10.3389/fcell.2020.00106

Xu, Z., Zeng, S., Gong, Z., and Yan, Y. (2020). Exosome-Based Immunotherapy: A Promising Approach for Cancer Treatment. Mol. Cancer 19 (1), 160. doi:10.1186/s12943-020-01278-3

Ye, Y., Dai, Q., and Qi, H. (2021). A Novel Defined Pyroptosis-Related Gene Signature for Predicting the Prognosis of Ovarian Cancer. Cell Death Discov. 7 (1), 71. doi:10.1038/s41420-021-00451-x

Yoshihara, K., Shahmoradgoli, M., Martínez, E., Vegesna, R., Kim, H., Torres-Garcia, W., et al. (2013). Inferring Tumour Purity and Stromal and Immune Cell Admixture from Expression Data. Nat. Commun. 4, 2612. doi:10.1038/ncomms3612

Yu, Z., Wang, J., Cai, X., Gao, Z., Wang, S., and Gu, Y. (2020). Analysis of Pemetrexed-Based Chemotherapy in the Treatment of Advanced Colorectal Cancer. Ann. Transl Med. 8 (21), 1368. doi:10.21037/atm-20-1095
Zaiachuk, M., Pryimak, N., Kovalchuk, O., and Kovalchuk, I. (2021). Cannabinoids, Medical Cannabis, and Colorectal Cancer Immunotherapy. Front. Med. (Lausanne) 8, 713153. doi:10.3389/fmed.2021.713153

Zhang, H., Li, L., and Liu, L. (2018). Fc $\gamma$ RI (CD64) Contributes to the Severity of Immune Inflammation through Regulating NF-Kb/nlrp3 Inflammasome Pathway. Life Sci. 207, 296-303. doi:10.1016/j.lfs.2018.06.015

Zhang, X., Gao, S., Li, Z., Wang, W., and Liu, G. (2020). Identification and Analysis of Estrogen Receptor a Promoting Tamoxifen Resistance-Related lncRNAs. Biomed. Res. Int. 2020, 9031723. doi:10.1155/2020/9031723

Zhang, L., Wang, Z., Li, M., Sun, P., Bai, T., Wang, W., et al. (2021). HCG18 Participates in Vascular Invasion of Hepatocellular Carcinoma by Regulating Macrophages and Tumor Stem Cells. Front. Cel Dev. Biol. 9, 707073. doi:10.3389/fcell.2021.707073

Zhao, D., Chen, H., and Wang, B. (2021). Assessing the Regulatory Functions of LncRNA SNHG11 in Gastric Cancer Cell Proliferation and Migration. Front. Cel Dev. Biol. 9, 620476. doi:10.3389/fcell.2021.620476

Zheng, J., Zhou, Z., Qiu, Y., Wang, M., Yu, H., Wu, Z., et al. (2021a). A Prognostic Ferroptosis-Related IncRNAs Signature Associated with Immune Landscape and Radiotherapy Response in Glioma. Front. Cel Dev. Biol. 9, 675555. doi:10.3389/ fcell.2021.675555

Zheng, Z., Zhang, Q., Wu, W., Xue, Y., Liu, S., Chen, Q., et al. (2021b). Identification and Validation of a Ferroptosis-Related Long Non-coding RNA Signature for Predicting the Outcome of Lung Adenocarcinoma. Front. Genet. 12, 690509. doi:10.3389/ fgene.2021.690509

Conflict of Interest: The authors declare that the research was conducted in the absence of any commercial or financial relationships that could be construed as a potential conflict of interest.

Publisher's Note: All claims expressed in this article are solely those of the authors and do not necessarily represent those of their affiliated organizations, or those of the publisher, the editors, and the reviewers. Any product that may be evaluated in this article, or claim that may be made by its manufacturer, is not guaranteed or endorsed by the publisher.

Copyright (C) $2021 \mathrm{Xia}$, Yan and Shen. This is an open-access article distributed under the terms of the Creative Commons Attribution License (CC BY). The use, distribution or reproduction in other forums is permitted, provided the original author(s) and the copyright owner(s) are credited and that the original publication in this journal is cited, in accordance with accepted academic practice. No use, distribution or reproduction is permitted which does not comply with these terms. 